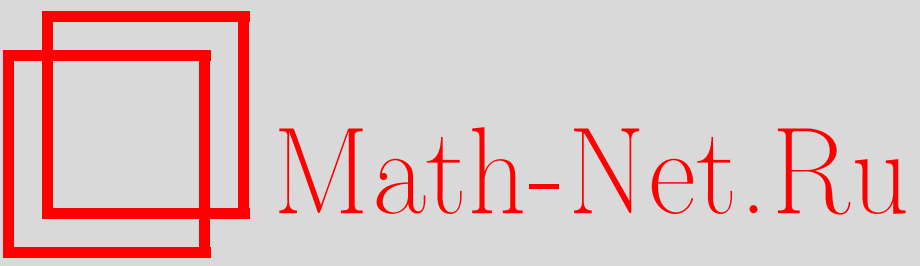

Д. В. Ширков, К фурье-преобразованию эффективной функции связи, ТМФ, 2003, том 136, номер 1, 3-19

DOI: https://doi.org/10.4213/tmf217

Использование Общероссийского математического портала Math-Net.Ru подразумевает, что вы прочитали и согласны с пользовательским соглашением

http://www.mathnet.ru/rus/agreement

Параметры загрузки:

IP: 3.89 .185 .249

26 апреля 2023 г., 14:08:41 
ТЕОРЕТИЧЕСКАЯ

И МАТЕМАТИЧЕСКАЯ

ФИЗИКА

Том 136, № 1

июль, 2003

(C) 2003 г.

Д.В. Ширков*

\section{К ФУРЬЕ-ПРЕОБРАЗОВАНИЮ ЭФФЕКТИВНОЙ ФУНКЦИИ СВЯЗИ}

Рассматриваются интегральные преобразования ренорминвариантной функции связи КХД. Особое внимание уделено преобразованию Фурье, т.е. переходу от пространственно-временной картины к представлению энергии-импульса. Наше первое заключение состоит в том, что условие возможности такого перехода представляет еще один аргумент против реальности существования нефизических сингулярностей, наблюденных в пертурбативной КХД. Второе сводится к способу “перевода" некоторых сингулярных "длинноволновых" асимптотик в инфракрасную область переданных импульсов, который следует проводить с учетом теоремы Таубера. Это замечание имеет отношение к недавним результатам по асимптотическому поведению эффективной функции связи KXД, полученным коллаборацией "ALPHA" на основе численных решеточных симуляций.

Ключевые слова: тауберова теорема, ИК-поведение в КХД, преобразование Фурье.

Посвящается 50-летию безвременно ушедиего Никиты Алексеевича Свешникова, моего ученика и друга

\section{1. ИНВАРИАНТНАЯ ФУНКЦИЯ СВЯЗИ В РАЗЛИЧНЫХ ПРЕДСТАВЛЕНИЯХ}

В современных квантово-полевых вычислениях, как правило, выражают наблюдаемые и некоторые другие вспомогательные, инвариантные (или ковариантные) относительно преобразований ренормализационной группы (РГ), объекты через инвариантную функиию связи $\bar{\alpha}\left(Q^{2}\right)$ (называемую также әффективной или бегущей константой связ $\left.{ }^{1)}\right)$, которая является действительной функцией действительного положительного аргумента $Q^{2} \equiv \mathbf{Q}^{2}-Q_{0}^{2}-$ квадрата переданного импульса.

Понятие инвариантной связи (ИС) первоначально было введено ${ }^{2)}$ (см., например, paботы [1], а также главу “Ренормализационная группа" в монографии [2]) в виде произ-

\footnotetext{
1) Ввиду явной семантической абсурдности последнего выражения мы используем термин инвариантная функция связи, а также инвариантная или эффективная связь.

${ }^{2)}$ С использованием термина "инвариантный заряд" (электрона), естественного в контексте КЭД.
}

* Лаборатория теоретической физики им. Н. Н. Боголюбова, Объединенный институт ядерных исследований, Дубна, Московская обл., Россия. E-mail: shirkovd@thsun1.jinr.ru 
ведения действительных констант $z_{i}$, входящих в конечные перенормировочные преобразования Дайсона

$$
D_{i}(\ldots, \alpha) \rightarrow D_{i}^{\prime}\left(\ldots, \alpha^{\prime}\right)=z_{i}^{-1} D_{i}\left(\ldots, z_{3} \alpha\right), \quad \alpha \rightarrow \alpha^{\prime}=z_{3} \alpha
$$

где пропагаторы $D_{i}(\ldots, \alpha)=D_{i}(Q, m, \mu ; \alpha)$ обычно берутся в импульсно-энергетическом представлении. Здесь ИС выражается через скалярные (т.е. лоренц-инвариантные) квантово-полевые амплитуды, взятые также в импульсном представлении. Так, например, в КЭД ИС

$$
\bar{\alpha}\left(Q^{2}\right) \equiv \alpha d\left(\frac{Q^{2}}{\mu^{2}}, \frac{m^{2}}{\mu^{2}} ; \alpha\right)
$$

где $d$ - поперечная амплитуда фотонного пропагатора, является действительной в евклидовой области. В КХД ИС $\bar{\alpha}_{s}\left(Q^{2}\right)$ обычно определена подобным образом в виде произведения параметра взаимодействия $\alpha_{s}$ и подходяших скалярных пропагаторных и вершинных амплитуд (см., например, работу [3] и приведенные в ней ссылки).

Эта ИС используется для параметризации ренормализационно-инвариантных величин, в том числе наблюдаемых. Для этого следует взять интересующий нас объект в соответствуюшем представлении. В частности, через $\bar{\alpha}_{s}\left(Q^{2}\right)$ можно выразить лишш величину, представленную в импульсном представлении. Более того, последняя, для того чтобы быть представимой через действительную функцию $\bar{\alpha}_{s}\left(Q^{2}\right)$, сама должна быть действительной, т.е. быть функцией переменной $Q^{2}>0$ в евклидовой области.

Между тем некоторые наблюдаемые, подобные эффективным поперечным сечениям рассеяния, зависят от другого лоренц-инвариантного аргумента $s$ - квадрата энергии в системе центра масс. Как хорошо известно, поляризационный оператор $\Pi\left(Q^{2}\right)$, представленный в виде интеграла Коши, доставляет нам техническую возможность интегральной связи между функциями переменных $Q^{2}$ и $s$. Его логарифмическая производная, известная как функция Адлера, определяется через интеграл

$$
D\left(Q^{2}\right)=Q^{2} \int_{0}^{\infty} \frac{d s}{\left(s+Q^{2}\right)^{2}} R(s),
$$

полученный дифференцированием спектрального представления Челлена-Лемана для $\Pi\left(Q^{2}\right)$. Здесь $R(s)$ - мнимая часть поляризационного оператора - в силу оптической теоремы пропорциональна полному эффективному поперечному сечению, являющемуся наблюдаемой величиной.

Ниже мы будем использовать соотношение (2) как основу для определения интегральной операции $\mathbb{D}$, преобразующей действительную функцию $M(s)$ положительного (времениподобного) аргумента в другую действительную функцию $E\left(Q^{2}\right)$ положительного действительного (пространственноподобного) аргумента $Q^{2}$ (см. ниже соотношение $(7))$.

Введем более обшее линейное интегральное преобразование с ядром $K(x)$, зависящим от одного аргумента:

$$
f(x) \rightarrow F(y)=\mathbb{L}[f](y)=\int_{0}^{\infty} d(x y) K(x y) f(x),
$$


и включающим различные виды одномерного преобразования Фурье (обычное, Лапласа, синус- и косинус-преобразования Фурье и некоторые другие, например с ядром $K(x) \sim J_{1}(x) x^{2}$, которое можно получить из 4-мерного преобразования Фурье) и, при $K(x)=\left(1+x^{2}\right)^{2}$, только что упомянутое преобразование $A$ длера $\mathbb{D}$, определенное ниже соотношением (7).

Раздел 2 мы начинаем с рассмотрения взаимоотношения между РГ-инвариантностью и интегральным преобразованием. Наш первый явный пример, взятый из так называемой аналитической теории возмущений (ATB), относится к переходу из минковской части импульсно-энергетического многообразия в соответствующую евклидову область. Затем рассматривается переход из представления расстояний (трехмерного пространства координат) в обшепринятое представление (переданных) импульсов с помощью одномерного синус-преобразования Фурье.

Далее в разделе 3 мы обращаем особое внимание на соотношение между асимптотическим поведением на больших расстояниях и инфракрасной (ИК) асимптотикой КХД, что приводит нас к замечанию по поводу возможной интерпретации в ИК-области некоторых недавних результатов коллаборации "ALPHA".

\section{2. ИНТЕГРАЛЬНЫЕ ПРЕОБРАЗОВАНИЯ}

2.1. РГ-инвариантность и интегральные преобразования. Прежде всего отметим, что преобразование $\mathbb{L}$ совместно с РГ-преобразованием. Так, например, пусть (в безмассовом случае) исходная функция $f(x / \mu ; \alpha)$ инвариантна по отношению к РГ-преобразованию

$$
R_{\tau}:\left\{\mu^{2} \rightarrow\left(\mu^{\prime}\right)^{2}=\mu^{2} \tau ; \quad \alpha \rightarrow \alpha^{\prime}=\bar{\alpha}_{\mathrm{tr}}(\tau, \alpha)\right\} .
$$

Тогда ее интегральный образ $F(y \mu ; \alpha)=\mathbb{L}[f](y)$ будет инвариантным по отношению к тому же самому РГ-преобразованию (4) - и наоборот - с той жсе самой функиией преобразования $\bar{\alpha}_{\mathrm{tr}}$, удовлетворяюшей функциональному уравнению

$$
\bar{\alpha}(\theta \tau, \alpha)=\bar{\alpha}(\theta, \bar{\alpha}(\tau, \alpha)),
$$

вытекаюшему из группового закона сложения $R_{\theta} R_{\tau}=R_{\theta \tau}$. Отметим, что из этого уравнения для функции $\bar{\alpha}_{\mathrm{tr}}$ следует каноническое условие нормировки

$$
\bar{\alpha}_{\mathrm{tr}}(1, \alpha)=\alpha
$$

отвечающее тождественному преобразованию $R_{1}$.

Соотношение (2) обычно рассматривается как определение функции Адлера $D$. Вместо этого, однако, мы будем считать его определением интегральной операции $\mathbb{D}$, преобразуюшей функцию $M(s)$ положительного действительного аргумента $s$ в другую функцию $E\left(Q^{2}\right)$ положительного действительного аргумента $Q^{2}$ :

$$
M(s) \rightarrow \mathbb{D}[M]\left(Q^{2}\right) \equiv Q^{2} \int_{0}^{\infty} \frac{d s}{\left(s+Q^{2}\right)^{2}} M(s)=E\left(Q^{2}\right) .
$$

При этом обратная операция $\mathbb{R}=[\mathbb{D}]^{-1}$ представима с помошью контурного интеграла (более подробно об этом см., например, в работах [4], [5]). 
Преобразования $\mathbb{D}$ и $\mathbb{R}$ сохраняют РГ-инвариантность. Так, например, они преобразуют ренормализационно-инвариантную функцию $M\left(s / \mu^{2} ; \alpha\right)$ в другую инвариантную функцию $E\left(Q^{2} / \mu^{2} ; \alpha\right)$ и наоборот. Первый из этих инвариантов $M$ должен быть представим в виде функции от подходящей “минковской” инвариантной связи $\alpha(s)$, тогда как другой инвариант $E$ - от обычной “евклидовой” функции связи $\bar{\alpha}\left(Q^{2}\right)$. Это, в частности, означает, что, отправляясь от обычной функции связи $\mathrm{KX} Д \bar{\alpha}_{s}\left(Q^{2}\right)$, посредством операции $\mathbb{R}$ можно определить ${ }^{3)}$ эффективную функцию связи КХД во времениподобной (минковской) области $\alpha_{M}(s)=\mathbb{R}\left[\bar{\alpha}_{s}\right](s)$ через $\bar{\alpha}_{s}\left(Q^{2}\right)$, инвариантную функцию связи КХД, первоначально определенную в евклидовой области.

В качестве другого примера возьмем физическую амплитуду $A$, зависящую от двух лоренш-инвариантных кинематических аргументов $s$ и $t$. Положим, что она также ренормализационно-инвариантна: $A(s, t ; \mu, \alpha)=A\left(s, t ; \mu^{\prime}, \alpha^{\prime}\right)$. Здесь возникают две возможности. Первая связана с интегральным преобразованием по отношению лишь к одному из аргументов, подобным преобразованию эйконала $F(s, t) \rightarrow \Phi(s, b)$. Новая амплитуда $\Phi$ имеет трансформационные РГ-свойства, подобные свойствам $F$. Как и в обшем случае нескольких кинематических переменных, РГ-свойства $F$ и $\Phi$ не оказываются особено полезными.

Однако положение меняется при интегральном преобразовании второго типа, вовлекаюшем функцию отношения обеих переменных $\varphi(s / t)$. Такое преобразование

$$
A(s, t) \rightarrow A_{k}(s)=\int_{0}^{\infty} A(s, t) K_{(k)}\left(\frac{s}{t}\right) d \varphi\left(\frac{s}{t}\right), \quad k=0,1,2, \ldots,
$$

отображает исходную функцию двух переменных на функцию (набор функций) $A_{k}(s)$ одного аргумента, которая(ые) является(ются) РГ-инвариантной(ыми). Проекции на парциальные волны и моменты структурных функций доставляют примеры такого рода.

Еще один пример интегрального преобразования “одноаргументной” функции, совместного с РГ-инвариантностью, дает преобразование Фурье, которое является частным случаем линейного преобразования (3). Оно связьвает функцию $f(r)$ пространственно-временного лоренц-инвариантного аргумента $r=\sqrt{\mathbf{r}^{2}-t^{2}}$ с функцией $F(Q)$ инварианта $Q=\sqrt{Q^{2}=\mathbf{Q}^{2}-Q_{0}^{2}}$ импульсно-энергетического представления.

Известно, что преобразование Дайсона (1), реализуюшее свойство самоподобия [6] уравнений Швингера-Дайсона, может, на равных основаниях с энергетически-импульсным (как в (1)), формулироваться также и в пространственно-временном представлении, т.е. для функции вида $D(\ldots, \alpha)=\widetilde{D}(x, m, \mu ; \alpha), x=\{\mathbf{r}, t\}$.

В контексте КЭД последнее представление ${ }^{4)}$ было использовано Дираком в $30-$ х годах при обсуждении эффектов поляризации вакуума в терминах әффективного заряда әлектрона [7]

$$
\bar{e}_{\mathrm{D}}(r)=e_{0}\left\{1-\frac{\alpha_{0}}{3 \pi} \ln \frac{r}{r_{e}}\right\}, \quad \alpha_{0}=\frac{e_{0}^{2}}{4 \pi} \simeq \frac{1}{137.0360}, \quad r_{e}=\frac{h}{m_{e} c},
$$

\footnotetext{
${ }^{3)}$ Однако на практике нефизические сингулярности пертурбативной $\bar{\alpha}_{s}\left(Q^{2}\right)$ препятствуют выполнению необходимого интегрирования. Более подробно этот вопрос обсуждается в работе [5].

4) Мы будем именовать его представлением расстояний.
} 
введенного им в виде пространственного распределения электрического заряда вокруг точечного "голого" электрона.

В наше время аналогичный объект - эффективная функция связи $\mathrm{KX} \bar{g}^{2}(L)$, являющаяся функцией пространственного размера решетки $L,-$ был введен [8] и использован [9]-[12] в работах коллаборации "АLPHA".

Некоторые другие примеры взаимоотношения между интегральными преобразованиями и РГ-симметриями в задачах классической и математической физики читатель может найти в недавнем обзоре Ковалева [13] (см., в частности, пример 2 на с. 358 и имеющиеся там ссылки).

Вопрос соответствия между формулировками РГ в различных представлениях имеет два аспекта. Один из них относится к основным объектам, т.е. к формулировке РГ-преобразований. Второй связан с корреляцией этих объектов через подходящие интегральные преобразования.

Согласно терминологии, используемой в разделе 48.5 книги [2] и в работе [14], инвариантную функцию, удовлетворяюшую функциональному уравнению (5) и каноническому условию нормировки, следует именовать эффективной связью (ЭС). Случаю инвариантной функции $\bar{\alpha}_{N}$ с более общим условием нормировки

$$
\bar{\alpha}_{N}(1, \alpha)=N(\alpha) \neq \alpha
$$

отвечает термин инвариантная связь. Последняя, в отличие от ЭС, не может использоваться в качестве функции преобразования константы связи в РГ-преобразовании (4). В общем случае интегральное преобразование (3) отображает ЭС $\bar{\alpha}(x)$ на некоторую РГ-инвариантную функцию $A(y)$, являюшуюся ИС, а не ЭС.

В этой связи возникают следуюшие вопросы:

1. Как явно определить $\bar{\alpha}$ и ее интегральньй образ $A$ для данной модели КТП?

2. Как выразить их друг через друга?

3. Какую из них можно использовать в роли функции РГ-преобразования $\alpha_{\mathrm{tr}}$ ?

"Немедленные" ответы:

1) использовать обычный алгоритм с пертурбативными бета-функциями для получения $\bar{\alpha}$ и $A$,

2) соотнести их с помошью подходяшего преобразования (3) - оказываются несовместимыми друг с другом.

2.2. От представления энергии в с.ц.м. к представлению переданного импульса. Эта ситуация может быть разъяснена с помощью известных ответов на те же самые вопросы для интегрального преобразования (7). Рассмотрим частный случай этого преобразования - преобразование функции связи

$$
\alpha(s) \rightarrow \mathbb{D}[\alpha]\left(Q^{2}\right) \equiv Q^{2} \int_{0}^{\infty} \frac{d s}{\left(s+Q^{2}\right)^{2}} \alpha(s)=\bar{\alpha}\left(Q^{2}\right) .
$$

Оказывается невозможным использовать обычную пертурбативную функцию связи КХД $\bar{\alpha}_{s}\left(Q^{2}\right)$ в качестве $\bar{\alpha}\left(Q^{2}\right)$ из-за ее нефизических сингулярностей, подобных полюсу Ландау при $Q^{2}=\Lambda^{2}$, наличие которых противоречит интегральному представлению (9). Последнее подразумевает, что функция $\bar{\alpha}\left(Q^{2}\right)$ является свободной от каких- 
либо сингулярностей вне разреза $0>Q^{2}>-\infty$. То же самое относится к кандидатуpe $\bar{\alpha}_{s}\left(Q^{2}\right)$ на роль функции $\alpha(s)$. Одно из возможных решений, предложенное в рамках ATB (см. [15], [5]), состоит в использовании $\bar{\alpha}_{s}\left(Q^{2}\right)$ только в качестве прототипа как для $\bar{\alpha}\left(Q^{2}\right)$, так и для $\alpha(s)$, которые в однопетлевом приближении при

$$
\bar{\alpha}_{s}^{(1)}\left(Q^{2}\right)=\frac{1}{\beta_{0} \ln \left(Q^{2} / \Lambda^{2}\right)}, \quad \Lambda^{2}=\mu^{2} e^{-1 /\left(\beta_{0} \alpha_{s}\right)},
$$

имеют вид

$$
\alpha^{(1)}(s)=\left.\frac{1}{\pi \beta_{0}} \arccos \frac{L_{s}}{\sqrt{L_{s}^{2}+\pi^{2}}}\right|_{L_{s}>0}=\frac{\operatorname{arctg}\left(\pi / L_{s}\right)}{\pi \beta_{0}}, \quad L_{s}=\ln \frac{s}{\Lambda^{2}},
$$

$$
\bar{\alpha}^{(1)}\left(Q^{2}\right)=\frac{1}{\beta_{0}}\left[\frac{1}{L}-\frac{\Lambda^{2}}{Q^{2}-\Lambda^{2}}\right], \quad L=\ln \frac{Q^{2}}{\Lambda^{2}} .
$$

Обе эти функции, соответствуюшие (10) в пределе слабой связи, являются свободными от нефизических сингулярностей монотонными функциями, связанными меж ду собой преобразованием $(9) \bar{\alpha}\left(Q^{2}\right)=\mathbb{D}[\alpha]\left(Q^{2}\right)$ и ему обратным.

Как было отмечено в работе [5], переход от выражения $(10)$ для $\bar{\alpha}_{s}^{(1)}\left(Q^{2}\right)$ к $\alpha(s)$ и $\bar{\alpha}\left(Q^{2}\right)$ может быть представлен как следствие преобразования от обычной константы связи $\mathrm{KXД} \alpha_{s}$ к новым константам $\alpha_{\mathrm{M}}$ и $\alpha_{\mathrm{E}}$ :

$$
\alpha_{s} \rightarrow \alpha_{\mathrm{M}}=\frac{1}{\pi \beta_{0}} \arccos \frac{1}{\sqrt{1+\pi^{2} \beta_{0}^{2} \alpha_{s}^{2}}}=\frac{1}{\pi \beta_{0}} \operatorname{arctg}\left(\pi \beta_{0} \alpha_{s}\right)
$$

$$
\alpha_{s} \rightarrow \alpha_{\mathrm{E}}=\alpha_{s}+\frac{1}{\beta_{0}}\left(1-e^{1 /\left(\beta_{0} \alpha_{s}\right)}\right)^{-1} .
$$

Тем самым переход от минковской функции связи $\alpha(s)$ к евклидовой $\bar{\alpha}\left(Q^{2}\right)$ можно представлять индуцированным следующим преобразованием константы связи:

$$
\alpha_{\mathrm{M}} \rightarrow \alpha_{\mathrm{E}}\left(\alpha_{\mathrm{M}}\right)=\frac{1}{\pi \beta_{0}} \operatorname{tg}\left(\pi \beta_{0} \alpha_{\mathrm{M}}\right)+\frac{1}{\beta_{0}} \frac{1}{1-e^{\pi \operatorname{ctg}\left(\pi \beta_{0} \alpha_{\mathrm{M}}\right)}}
$$

при $0<\alpha_{\mathrm{M}} \leqslant 1 / \beta_{0}$ и $0<\alpha_{\mathrm{E}} \leqslant 1 / \beta_{0}$.

В свою очередь, отсюда следует, что интегральное преобразование (9) действительно изменяет нормировку функции связи и, в частности, отображает ЭС на ИС.

2.3. Импульсное представление и представление расстояний. Обратимся теперь к преобразованию Фурье. Нам окажется удобным использовать модифицированное синус-преобразование Фурье ${ }^{5)}$ вида

$$
\mathbb{F}_{\sin / r}[f](Q) \equiv \frac{2}{\pi} \int_{0}^{\infty} \frac{d r}{r} \sin (Q r) f(r)=F(Q),
$$

\footnotetext{
5) Оно является частным случаем преобразования (3) при $K(z)=2 \sin z /(\pi z)$, а также вытекает из обычного трехмерного преобразования

$$
\bar{\psi}(Q)=(2 \pi)^{-2} \int d \mathbf{r} \psi(r) e^{i \mathbf{Q r}} \text { при } F(Q)=Q^{2} \bar{\psi}(Q), \quad f(r)=r \psi(r) .
$$
}


совместное с РГ-преобразованием, а также ему обратное

$$
\mathbb{F}_{\sin / r}^{-1}[F](r) \equiv r \int_{0}^{\infty} d Q \sin (Q r) F(Q)=f(r)
$$

Вернемся теперь к выражению (8), возведя его в квадрат и переписав в более современных и общих обозначениях:

$$
\alpha_{\mathrm{D}}^{\mathrm{pt}}\left(\frac{r^{2}}{r_{\mu}^{2}}, \alpha_{\mu}\right)=\alpha_{\mu}\left\{1-\frac{\alpha_{\mu}}{3 \pi} \ln \frac{r^{2}}{r_{\mu}^{2}}\right\} .
$$

Здесь $r_{\mu}$-некоторое отсчетное расстояние, входящее в соотношение соответствия: $\alpha_{\mu}=$ $\alpha_{0}$ при $r_{\mu}=r_{e}$. Эта пертурбативная ЭС в представлении расстояний $\alpha_{\mathrm{D}}^{\mathrm{pt}}\left(\rho^{2}, \alpha\right)$ может быть связана преобразованием

$$
\bar{\alpha}_{\mathrm{pt}}\left(\frac{Q^{2}}{\mu^{2}}, \alpha\right)=\mathbb{F}_{\sin / r}\left[\alpha_{\mathrm{D}}^{\mathrm{pt}}\right]\left(Q^{2}\right)
$$

с более привычной пертурбативной функцией связи КЭД в импульсном представлении

$$
\bar{\alpha}_{\mathrm{pt}}\left(\frac{Q^{2}}{\mu^{2}}, \alpha_{\mu}\right)=\alpha_{\mu}\left\{1+\frac{\alpha_{\mu}}{3 \pi} \ln \frac{Q^{2}}{\mu^{2}}\right\}
$$

где $\mu=c_{\mathrm{E}} / r_{\mu}, c_{\mathrm{E}}=e^{-\mathbf{C}}=0.5614$. Выражение (16) может быть также получено из (15) заменой аргумента

$$
r \rightarrow \frac{c_{\mathrm{E}}}{Q}
$$

которая близка к соотношению квантово-механического соответствия $r \rightarrow 1 / Q$, отличаясь от него лиш корректировкой шкалы $Q$. Заметим, что положительньй эффект корректировочного фактора $c_{\mathrm{F}}$ четко виден на рис. 2 (см. ниже).

В полной аналогии с представленным выше примером обе функции $\bar{\alpha}^{\mathrm{RG}}$ и $\alpha_{\mathrm{D}}^{\mathrm{RG}}$, будучи явно определены (в знакомой форме суммы геометрической прогрессии) обычной РГ-процедурой с использованием выражений (15) и (16) для нахождения бета-функций, не связаны между собой преобразованием (14). Вместо этого они оказываются связанными более сложным соотношением $\bar{\alpha}^{\mathrm{RG}}(Q)=\Psi\left\{\mathbb{F}_{\sin }\left[\alpha_{\mathrm{D}}^{\mathrm{RG}}\right](Q)\right\}$.

Более того, вообще говоря, преобразование Фурье отображает ЭС на некоторую инвариантную функцию связи, которая не может быть использована как $\alpha_{\operatorname{tr}}$ в (5). Так, например, отправляясь от пертурбативной ЭС в представлении расстояний

$$
\alpha_{\mathrm{D}}^{\mathrm{pt}}\left(\rho^{2}, \alpha\right)=\alpha-\beta(\alpha) \ln \rho^{2}+\alpha\left(\frac{\alpha}{3 \pi}\right)^{2} \ln ^{2} \rho^{2}, \quad \rho^{2}=\frac{r^{2}}{r_{\mu}^{2}}, \quad \alpha=\alpha_{\mu},
$$

в результате использования синус-преобразования Фурье (14) получим

$$
\bar{\alpha}_{\mathrm{pt}}\left(q^{2}, \alpha\right)=\alpha+\beta(\alpha) \ln q^{2}+\alpha\left(\frac{\alpha}{3 \pi}\right)^{2}\left\{\ln ^{2} q^{2}+\Delta_{2}\right\}, \quad q^{2}=\frac{Q^{2}}{\mu^{2}}=\left(\frac{Q r_{\mu}}{c_{\mathrm{E}}}\right)^{2},
$$


причем $\Delta_{2}=\pi^{2} / 12$. Поэтому $\bar{\alpha}(1, \alpha)=\alpha+\alpha(\alpha /(3 \pi))^{2} \Delta_{2}=\alpha^{\prime} \neq \alpha$ и $\bar{\alpha}\left(q^{2}, \alpha\right)$ представляет собой ИС ${ }^{6)}$. Переходом к другой константе связи $\alpha \rightarrow \alpha^{\prime}$ она может быть преобразована (см. раздел 3 работы [14]) в ЭС $\bar{\alpha}^{\prime}\left(q^{2}, \alpha^{\prime}\right)$. Это обстоятельство может оказаться существенным в случае сильной связи в ИК-области.

Отметим также, что логарифмические члены в (18) и (19) приводят к суммам геометрических прогрессий с аргументами логарифмов, связанными соотношением (17).

\section{3. АСИМПТОТИКА БОЛЬШИХ РАССТОЯНИЙ И ИК-АСИМПТОТИКА}

3.1. Тауберова теорема. Обратимся теперь к частному вопросу о степени корреляции между асимптотиками функций $f$ и $F$, связанных преобразованием Фурье.

Использование подобных корреляций обычно в квантовой механике, где зачастую применяют правило квантово-механического соответствия

$$
r \rightarrow \frac{1}{Q}
$$

которое в ИК-пределе эквивалентно условию

$$
F(Q) \sim f\left(Q^{-1}\right) \text { при } Q \rightarrow 0 .
$$

Последнее свойство может быть эвристически понято путем замены переменной интегрирования $r \rightarrow x=r Q$ общего линейного преобразования (3):

$$
F(Q)=\int_{0}^{\infty} \frac{d x}{x} K(x) f\left(\frac{x}{Q}\right) .
$$

Однако для более строгого вывода соотношения (20) следует конкретизировать асимптотическое поведение функции $f(r)$ при $r \rightarrow \infty$, наложив на него некоторое ограничение. Упрошая ситуацию, можно сформулировать следуюшую тауберову теорему (здесь символ “ " означает "ведет себя подобно" $)^{7)}$ :

Теорема. Если функиия $f(r)$ асимптотически удовлетворяет условию сепарабельности

$$
f(k r) \sim C \phi(r) \varrho(k) \quad n p u \quad k \rightarrow \infty \quad u \quad C \neq 0,
$$

тогда, при выполнении некоторых дополнительных условий, ее фурье-образ обладает свойством

$$
F(Q) \sim \varrho\left(\frac{1}{Q}\right) \quad \text { при } \quad Q \rightarrow 0 .
$$

\footnotetext{
6) Отметим, что это обстоятельство оказывается несущественным (так же как и для преобразования (13)) в случае слабой связи на однопетлевом и двухпетлевом уровнях.

7) Первоначально тауберовыми теоремами назывались утверждения, связывающие свойства суммируемости и сходимости рядов. Позднее, в середине ХХ столетия, этот термин стал использоваться применительно к асимптотическим свойствам функций, связанных интегральными преобразованиями. Мы приводим лиш упрощенную формулировку этой важной теоремы для случая преобразования типа Фурье, ее набросок, достаточный для наших приложений. Более полное и строгое изложение этого материала читатель может найти в главе 3 монографии [16] (см. также теорему 4 в разделе 14 работы [17]).
} 
Последнее свойство, с некоторыми оговорками, следует из уравнения (3a).

Теперь, для некоторого допустимого класса функций $\varrho(k)$, удовлетворяющих условию (S), например для степенных и/или логарифмических функций

$$
f(r) \sim \varrho(r) \sim r^{\beta}(\ln r)^{\gamma}(\ln \ln r)^{\delta} \ldots, \quad r \rightarrow \infty,
$$

оказывается возможным получить из (Т) правило соответствия (20).

3.2. Примеры. Рассмотрим несколько простых примеров, приведенных в таблице.

\begin{tabular}{|l||c|c||c|c|}
\hline & $f(r)$ & $F(Q)=\mathbb{F}_{\sin / \mathrm{r}}[f]$ & $S_{\mathrm{LR}}^{f}$ & $(20)_{\mathrm{IR}}$ \\
\hline$\{*\}$ & $r F(r)$ & $\frac{2 f(Q)}{\pi Q}$ & $r \rightarrow \infty$ & $Q \rightarrow 0$ \\
\hline$\{1\}$ & $r^{\nu}, 0 \leqslant \nu<1$ & $\frac{2}{\pi \nu} \sin \left(\frac{\pi \nu}{2}\right) \Gamma(1+\nu) Q^{-\nu}$ & + & + \\
\hline$\{2\}$ & $\ln r$ & $\ln Q^{-1}-\mathbf{C}, \mathbf{C}=0.5772$ & + & + \\
\hline$\{3\}$ & $(\ln r)^{n}, n \geqslant 2$ & $\left(\ln Q^{-1}-\mathbf{C}\right)^{n}+\Delta_{n}$ & + & + \\
\hline$\{4\}$ & $\frac{r^{2}}{r^{2}+a^{2}}$ & $e^{-a Q}, a \geqslant 0$ & + & + \\
\hline$\{5\}$ & $e^{-a r}, a \geqslant 0$ & $\frac{2}{\pi} \operatorname{arctg}\left(\frac{Q}{a}\right)$ & - & - \\
\hline
\end{tabular}

В двух правых колонках отмечено (не)соответствие поведения функции $f(r)$ при $r \rightarrow$ $\infty$ условию (S) тауберовой теоремы, а также (не)выполнение условия (QMC) лишь в ИК-области в виде (20). При этом символы “+” и “-” оказываются полностью коррелированными.

Как следует из приводимой таблищы, в соответствии с (21) класс допустимых функций оказывается довольно узким. Он не включает, например, тригонометрические и показательные $\sim e^{a r}$ функции. Только функция из первой строки $F_{\{1\}}$ отвечает (с точностью до множителя!) правилу (QMC) на всем интервале изменения аргумента. Уже выражение из второй строки $F_{\{2\}}=\ln \left(Q^{-1}\right)-\mathbf{C}$ (где $\mathbf{C}$ - постоянная Эйлера), удовлетворяющее условию тауберовой теоремы при $Q \rightarrow 0$, вообще говоря, отличается от $f_{\{2\}}(1 / Q)$, особенно в области $Q \simeq 1$. То же самое относится к строке $\{3\}$, где константа $\Delta_{2}$ уже встречалась выше в (19).

Следующая строка $\{4\}$ содержит пример функции, удовлетворяющей тауберову условию $(\mathrm{S})$, но сушественно нарушаюшей правило (QMC) для поведения функции $F_{\{4\}}$ в целом. Сравнение поведения фурье-образа $F_{\{4\}}(Q)=e^{-a Q}$ c "QMC-преобразованной" исходной функцией $f_{\{4\}}(1 / Q)=\left(1+a^{2} Q^{2}\right)^{-1}$ оказывается весьма поучительным. Как видно на рис. 1 , относительная погрешность, будучи уже при $a Q \geqslant 0.2$ величиной порядка $15 \%$, быстро возрастает и при $a Q \simeq 1$ достигает уровня $30 \%$.

Лишь функция $f_{\{5\}}$ не удовлетворяет тауберовой теореме, несмотря на то что предельные значения $f_{\{5\}}(\infty)=F_{\{5\}}(0)=0$ совпадают.

Для того чтобы приблизиться к более реалистическому для КХ Д случаю, рассмотрим синус-преобразование Фурье (14) для класса функций $f(Q)$, удовлетворяюших спектральному представлению Челлена-Лемана

$$
f(q) \rightarrow F(r)=\frac{2}{\pi} \int_{0}^{\infty} \frac{d Q}{Q} \sin (Q r) \int_{0}^{\infty} \frac{d \sigma \rho(\sigma)}{\sigma+Q^{2}}
$$




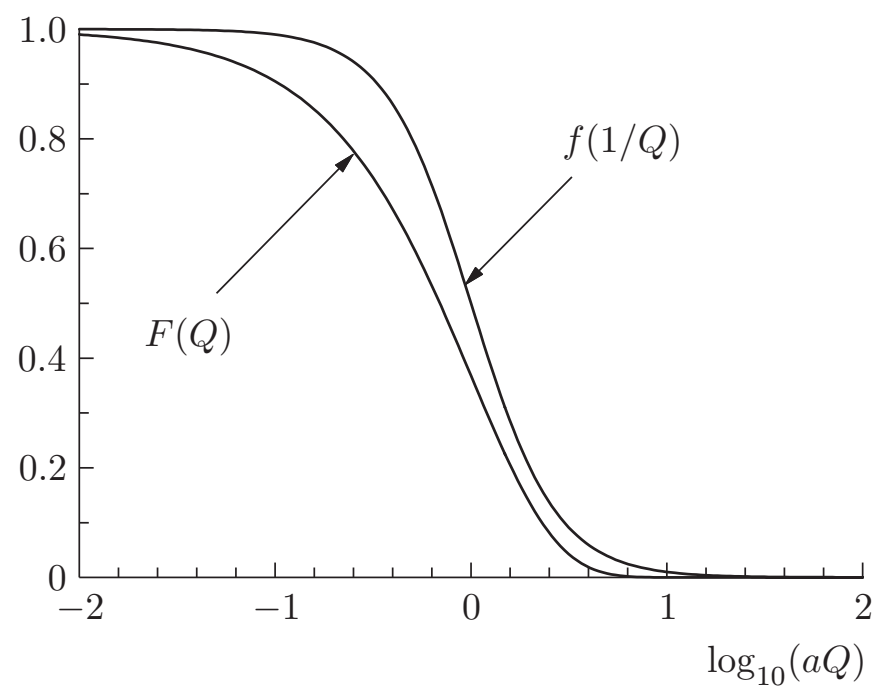

Рис. 1. Поведение "QМС-преобразованной" функции $f_{\{4\}}(1 / Q)=\left(1+a^{2} Q^{2}\right)^{-1}$ в сравнении с ее синус-фурье-образом $F_{\{4\}}(Q)=e^{-a Q}$.

со спектральной плотностью $\rho(\sigma)$. Меняя порядок интегрирования и интегрируя по $Q$ с помошью строки $\{4\}$ из таблицы, получаем

$$
F(r)=\int_{0}^{\infty} \frac{\rho(\sigma) d \sigma}{\sigma}\left(1-e^{-r \sqrt{\sigma}}\right) .
$$

В случае положительной спектральной плотности это выражение определяет монотонно возрастающую функцию $r$ с возможной сингулярностью при $r \rightarrow \infty$.

В качестве конкретного примера рассмотрим "аналитизированную" функцию связи $\mathrm{KX}$ Д $\bar{\alpha}\left(Q^{2}\right)$ (соотношение (12) со спектральной плотностью $\left.\rho_{\mathrm{APT}}(\sigma) \sim\left[(\ln \sigma)^{2}+\pi^{2}\right]^{-1}\right)$, взятую из однопетлевого приближения АТВ [18]. Для $a_{\mathrm{APT}}=\beta_{0} \alpha_{\mathrm{APT}}$ это дает

$$
a_{\mathrm{APT}}(r)=\int_{0}^{\infty} \frac{d \sigma\left(1-e^{-r \sqrt{\sigma}}\right)}{\sigma\left[(\ln \sigma)^{2}+\pi^{2}\right]}=1-I(r), \quad I(r)=\int_{0}^{\infty} \frac{e^{-r \sqrt{\sigma}} d \sigma}{\sigma\left[(\ln \sigma)^{2}+\pi^{2}\right]} .
$$

Интеграл в правой части $I(r)$ напоминает интеграл Рамануджана (см. главу 18.3 в книге [19])

$$
R(r) \equiv \int_{0}^{\infty} \frac{e^{-r x} d x}{x\left[(\ln x)^{2}+\pi^{2}\right]}=2 \int_{0}^{\infty} \frac{e^{-r \sqrt{\sigma}} d \sigma}{\sigma\left[(\ln \sigma)^{2}+4 \pi^{2}\right]}=\nu(r)-e^{r},
$$

выражаемьй через специальную трансцендентную функцию $\nu(x)$. Мы используем эту близость для анализа поведения $I(r)$ при $r \rightarrow 0$. Асимптотика функции $\nu$ хорошо изучена [19]. Получаем

$$
R(r) \rightarrow-1+\frac{1}{\ln r^{2}}+O\left(\frac{1}{\ln ^{2} r^{2}}\right) \text { при } r \rightarrow 0 .
$$


Разность

$$
\Delta(r)=I(r)-\frac{R(r)}{2}=3 \pi^{2} \int_{0}^{\infty} \frac{d \sigma}{\sigma} \frac{e^{-r \sqrt{\sigma}}}{\left[(\ln \sigma)^{2}+\pi^{2}\right]\left[(\ln \sigma)^{2}+4 \pi^{2}\right]}
$$

будучи положительной, исчезает на бесконечности, $\Delta(\infty)=0$. В то же время $\Delta(0)=$ 1/4. Поэтому

$$
a_{\mathrm{APT}}(r) \rightarrow \frac{1}{\ln r^{2}} \quad \text { при } \quad r \rightarrow 0 ; \quad a_{\mathrm{APT}}(\infty)=1 .
$$

Интересно сравнить монотонную исходную функцию

$$
\begin{gathered}
\bar{a}_{\mathrm{an}}\left(Q^{2}\right)=\beta_{0} \bar{\alpha}\left(Q^{2}\right)=\int_{0}^{\infty} \frac{\rho_{\mathrm{APT}}\left(w^{2}\right) d w^{2}}{w^{2}+q^{2}}=\frac{1}{\ln q^{2}}+\frac{1}{1-q^{2}}, \\
a_{\mathrm{an}}(0)=1, \quad a_{\mathrm{an}}\left(Q^{2}\right) \rightarrow \frac{1}{\ln q^{2}} \quad \text { при } q^{2} \equiv \frac{Q^{2}}{\Lambda^{2}} \rightarrow \infty
\end{gathered}
$$

c ее фурье-образом $a_{\mathrm{D}}$ в координатах $r=1 / Q$.

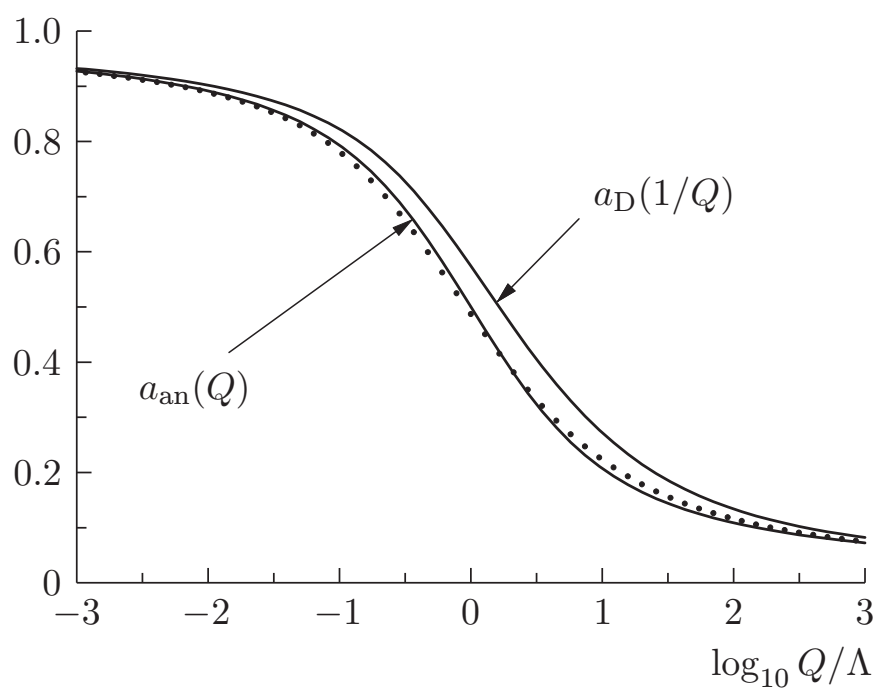

Рис. 2. Поведение АТВ-аналитизированной функции связи $\mathrm{KX}$ Д $a_{\mathrm{an}}(Q)$ в сравнении с ее фурье-образом $a_{\mathrm{D}}(1 / Q)$, в котором произведена замена аргумента по правилу (QMC). Пунктирная линия представляет фурье-образ $a_{\mathrm{D}}\left(c_{\mathrm{F}} / Q\right)$ с модификацией шкалы согласно (17).

Такое сравнение (см. рис. 2) выявляет удивительное подобие функций $a_{\text {an }}\left(Q^{2}\right)$ и $a_{\mathrm{D}}(1 / Q)$, которое значительно улучшается при замене $a_{\mathrm{D}}(1 / Q)$ на $a_{\mathrm{D}}\left(c_{F} / Q\right)$. В частности, максимальная ошибка, которая для первого случая достигает $30 \%$ в области $4 \lesssim Q / \Lambda \lesssim 70$, во втором случае не превышает $10 \%$. Тем самым возникает впечатление, что этот пример представляет собой сильный довод в пользу полезности правила (QMC) и (17) для качественной оценки фурье-образа функций с "правильными", т.е. соответствующими условию (21), асимптотиками. 
K сожалению, это впечатление обманчиво. Так, например, изучение семейства функций

$$
f\left(q^{2}\right)=\frac{1}{\ln ^{2}\left(q^{2}\right)+b^{2}}, \quad q^{2}=\frac{Q^{2}}{\Lambda^{2}},
$$

с "правильными" асимптотиками показывает ${ }^{8)}$, что отмеченное на рис. 2 подобие является скорее всего удивительным исключением из общей картины, которая, как правило, не дает даже качественного подобия типа, изображенного на рис. 1.

Таким образом, правило (QMC) в общем случае не является разумной основой для качественной оценки поведения фурье-образа даже для функции, удовлетворяющей условию тауберовой теоремы.

3.3. Функция связи КХД-функционала Шредингера. Для получения непертурбативной информации об ИК-поведении группа "ALPHA" изучает KXД (как в бескварковом варианте, так и с двумя безмассовыми кварковыми ароматами) на решетке и использует для решеточных симулящий так называемый функиионал IIредингера, определенный на 4-мерном евклидовом пространственно-временном многообразии специальным образом: по трем пространственным измерениям наложены циклические граничные условия, тогда как “временная" координата выделена - значения калибровочных полей на “верхней” и “нижней” крышках отличаются на фазовый фактор, содержаший параметр $\eta$. Перенормированная эффективная функция связи $\alpha_{\mathrm{SF}}$ определена через производную $\Gamma^{\prime}=\partial \Gamma / \partial \eta$ эффективного действия, представленного в виде разложения $\Gamma=\alpha^{-1} \Gamma_{0}+\Gamma_{1}+\alpha \Gamma_{2}+\cdots$, как функция пространственного размера $L$ многообразия (см. уравнение (2.43) в работе [8] и (8.3) в [10]) $\alpha_{\mathrm{SF}}(L)=\Gamma_{0}^{\prime} / \Gamma^{\prime}$, т.е. как функиия в координатном представлении.

Полученные на этой основе недавние результаты выявляют чрезвычайно быстрый рост $\alpha_{\mathrm{SF}}(L)$ в области $L \rightarrow \infty$ при $\alpha_{\mathrm{SF}} \simeq 1$. Аналитическая аппроксимация [12] для результатов численных расчетов поведения $\alpha_{\mathrm{SF}}$ имеет экспоненциальную форму

$$
\alpha_{\mathrm{SF}}(L) \simeq e^{M L}
$$

где $M \simeq 2.3 / L_{\max }$, а $L_{\max }$ - точка отсчета в области достаточно слабой связи, неявно определяемая условием $\alpha_{\mathrm{SF}}\left(L_{\max }\right)=0.275$, что дает $L_{\max } \sim 1 / m_{\tau}$. При обсуждении ИК-поведения КХД (т.е. области $Q^{2} \rightarrow 0$ ) в работах группы “ALPHA" используется "обычное квантово-механическое" отождествление в виде

$$
L \rightarrow \frac{1}{\mu}
$$

причем $\mu$ практически рассматривается как импульс нормировки. Такой способ перехода от представления расстояний к импульсному эквивалентен рассмотренному в п. 3.1. Он хорошо работает в УФ-области, поскольку поведение $\sim \ln ^{-1} Q^{2}$ совместно с тауберовой теоремой. Тем не менее в прецизионных оценках, как, например, в оценках связи различных шкал $[9]^{9)}$, следует принимать во внимание модификацию шкалы (17). В то

\footnotetext{
8) Частное сообщение А. В. Нестеренко

${ }^{9)}$ На эту важную работу наше внимание было обрашено д-ром У. Волффом, которому автор выражает признательность.
} 
же время быстро возрастающая функция типа (28) не удовлетворяет условию (S) этой теоремы. Поэтому соответствие с ИК-поведением (т.е. при $Q^{2} \rightarrow 0$ ) требует более подробного анализа.

3.4. Представление результатов группы "ALPHA" в ИК-области. Вообще говоря, возможны две разные точки зрения при обсуждении перехода от результатов численных симуляций для функционала Шредингера, проведенных на конечной решетке размером $L$. С одной стороны, можно считать, что полученные численные результаты и их аналитическая аппроксимация (28) представляют собой основу для некоторого приближения к предельной, непрерывной функции $\alpha_{\mathrm{SF}}(L)$, которая может быть отображена с помошью преобразования Фурье. С другой стороны, поскольку на пространственных границах решетки наложены периодические граничные условия, можно полагать, что ряд Фурье более адекватен, нежели интеграл Фурье.

Ниже мы попытаемся построить фурье-образ в ИК-области для функции $\alpha_{\mathrm{SF}}(L)$, имея в виду обе эти возможности.

Для того чтобы приступить к выполнению интегрирования Фурье, представим полученную коллаборацией “ALPHA” функцию связи в представлении расстояний $\alpha_{\mathrm{SF}}$ в виде суммы двух слагаемых

$$
\alpha_{\mathrm{SF}}(L)=\alpha_{\mathrm{PT}}(L)+\alpha_{\mathrm{AL}}(L),
$$

где $\alpha_{\mathrm{PT}}$ - пертурбативный вклад, а $\alpha_{\mathrm{AL}}$ - сушественно непертурбативная часть.

Поскольку результаты всех непертурбативных вычислений в КХД - как численных симулящий на решетке, так и решений уравнений Швингера-Дайсона (УШД) - не обнаруживают каких-либо следов нефизических сингулярностей, мы, с одной стороны, заменим первый пертурбативный член $\alpha_{\mathrm{PT}}$ на некоторое регулярное выражение $\alpha_{\mathrm{PT}-\mathrm{R}}$, например на ATB-аналитизированное (24), или на выражение, подобное таковому из “модели с массивными глюболами" [20] либо полученному из решения УШД [21].

С другой стороны, мы аппроксимируем второе, непертурбативное слагаемое выражением, близким к (28), которое поддается дальнейшему интегрированию:

$$
\alpha_{\mathrm{AL}}(L) \simeq \alpha_{k}(L)=C_{k} \frac{\pi}{2}\left(\frac{L}{L_{m}}\right)^{k} e^{m\left(L-L_{m}\right)} .
$$

Здесь $k \geqslant 1$ - небольшое целое число, а значения параметров $m L_{m} \simeq 2.0, m$ и $\mathrm{C}_{k}$ следуют из решеточных аппроксимаций. Так, например, $C_{1} \simeq 0.013$.

Итак,

$$
\alpha_{\mathrm{SF}}(L)=\alpha_{\mathrm{PT}-\mathrm{R}}(L)+\alpha_{k}(L) .
$$

Для фурье-образа регуляризованной $\alpha_{\mathrm{PT}-\mathrm{R}}$ мы предположим поведение, связанное с исходной функцией подстановкой (QMC):

$$
\bar{\alpha}_{\mathrm{PT}-\mathrm{R}}(Q) \equiv \mathbb{F}_{\sin }\left[\alpha_{\mathrm{PT}-\mathrm{R}}\right](Q) \simeq \alpha_{\mathrm{PT}-\mathrm{R}}\left(\frac{1}{Q}\right),
$$

с конечным ИК-пределом, подобным (27),

$$
\bar{\alpha}_{\mathrm{PT}-\mathrm{R}}(0)=C, \quad 0<C<\infty .
$$


Такое предположение выглядит вполне правдоподобным в свете АТВ-примера из п. 3.2 (см. рис. 2).

Для проведения интегрирования $(14)$ с функцией $\alpha_{k}(L)$ необходимо прибегнуть к регуляризации. Для этого в подынтегральное выражение в правой части (14) мы вводим фактор обрезания ${ }^{10)}$

$$
\exp \left\{(m+\mu) \theta\left(L-\xi L_{m}\right)\left(\xi L_{m}-L\right)\right\}, \quad \xi=2.5 \div 3, \quad \mu \geqslant 0,
$$

с параметрами $\xi$ и $\mu$, регулирующими "место включения" и интенсивность обрезания.

В итоге искомая функция связи группы “ALPHA” в импульсном представлении выражается в виде суммы трех членов

$$
\bar{\alpha}_{\mathrm{SF}}(Q)=\bar{\alpha}_{\mathrm{PT}-\mathrm{R}}(Q)+\bar{\alpha}_{k, \mathrm{reg}}(Q)+\bar{\alpha}_{k, \operatorname{sing}}(Q ; \mu),
$$

причем первый из них определен формулой (31), а остальные могут быть получены (дифференцированием по параметру $m$ ) из выражений, соответствующих случаю $k=1$ в (29) при

$$
\bar{\alpha}_{1, \mathrm{reg}}(Q)=\frac{C_{1}}{L_{m}} \int_{L_{m}}^{\xi L_{m}} d L \sin (Q L) e^{m L}=\frac{C_{1}}{L_{m}}\left[f_{1}\left(Q, \xi L_{m}\right)-f_{1}\left(Q, L_{m}\right)\right]
$$

И

$$
\bar{\alpha}_{1, \text { sing }}(Q ; \mu)=e^{m(\xi-1) L_{m}} \frac{C_{1}}{L_{m}} \int_{\xi L_{m}}^{\infty} d L \sin (Q L) e^{\mu\left(\xi L_{m}-L\right)}=\frac{C_{1}}{L_{m}} \varphi_{1}\left(Q, \xi L_{m} ; \mu\right),
$$

где

$$
f_{1}(Q, L)=e^{m L} \frac{m \sin (Q L)-Q \cos (Q L)}{m^{2}+Q^{2}}, \quad \varphi_{1}(Q, L ; \mu)=e^{m L} \frac{Q \cos (Q L)+\mu \sin (Q L)}{\mu^{2}+Q^{2}} .
$$

Для периодической функции в результате преобразования возникает ряд, а не интеграл Фурье, и последний член в (33) отсутствует.

Наиболее важной качественной чертой функций $f_{k}$ и $\varphi_{k}$ является их ИК-поведение. Все эти функции стремятся к нулю линейно по $Q \rightarrow 0$. Исключение возникает лишь при $\mu=0$, когда появляется степенная сингулярность $\varphi_{k}(Q \rightarrow 0, \ldots) \rightarrow 1 / Q$.

Комбинируя этот результат с (32), заключаем, что функция $\bar{\alpha}_{\mathrm{SF}}(Q)$ в ИК-асимптотике может иметь либо конечный предел, либо полюс первого порядка (последнее только в случае интегрального преобразования Фурье), в полной противоположности с экспоненциальным ростом типа $\sim e^{m / Q}$, на мысль о котором наводят результаты коллаборации "ALPHA" (мы имеем в виду рис. 3 и 4 из работы [12]).

Отметим, что с более общей точки зрения экспоненциальный рост по $L$ является наиболее сильным из возможных для $\alpha(L)$. В частности, при $\ln \alpha(L) \sim L^{\nu}, \nu>1$, не имеется известных математических средств для выполнения преобразования Фурье. И наоборот, для ИК-асимптотики $\sim e^{m / Q}$ невозможно совершить обратное преобразование Фурье и “возвратиться" к $L$-представлению.

\footnotetext{
${ }^{10)}$ Строго говоря, такая экспоненциальная регуляризация соответствует обобщенному преобразованию Фурье (см., например, раздел 1.3 в книге [22]).
} 


\section{4. ОБСУЖ ДЕНИЕ}

Наше первое наблюдение относится к вопросу о сушествовании нефизических сингулярностей у эффективной функшии связи КХДи у некоторых других величин, подобных амплитудам пропагаторов. Эти сингулярности несовместны с интегральными преобразованиями. В частности, известная полюсная особенность при $Q \sim \Lambda \simeq 300-400$ ГэВ у обычной пертурбативной функции связи $\mathrm{KX}$ Д $\bar{\alpha}_{s}\left(Q^{2}\right)$ препятствует процедуре интегрирования, необходимой для выполнения преобразования Фурье. Это дает нам еше один теоретический аргумент против существования нефизических сингулярностей в физически содержательных теориях, подобных КЭД и КХД. Напомним, что все непертурбативные вычисления в КХД - как с помошью численных симуляций на решетке, так и на основе решения приближенных УШД - не дают нам каких-либо намеков на наличие нефизических сингулярностей.

Второй результат, основанный на анализе свойств преобразования Фурье, имеет отношение к квантово-механическому правилу соответствия (QMC), связываюшему асимптотические поведения функции $f(r)$ при $r \rightarrow \infty$ и ее фурье-образа $F(Q)$ при $Q \rightarrow 0$. Было показано, что это правило, являясь разумным для некоторого допустимого класса асимптотик (степенных и логарифмических), имеет свои жесткие пределы применимости.

Во-первых, даже для функции с допустимой асимптотикой правило (QMC) в области далекой от сингулярности приводит лишь к качественному соответствию, как это видно на рис. 1. Во-вторых, оно совершенно неверно для широкого класса асимптотик (нарушаюших условия тауберовой теоремы (S)), включаюших экспоненциальные.

В частности, это относится к экспоненциально растушей вместе с размером решетки $L$ эффективной функции связи КХД

$$
\alpha_{\mathrm{SF}}(L) \sim e^{m L},
$$

наблюденной в работах коллаборации "ALPHA" на основе численных решеточных симуляций для функционала Шредингера, которая согласно анализу п. 3.4 в представлении переданного импульса может соответствовать либо

конечному, либо

(ALPHA-IRa)

"слабо сингулярному" $1 / Q$

поведению в ИК-асимптотике. Поэтому упомянутые результаты, будучи правильно перенесены в импульсное представление, в ИК-области могут оказаться качественно близкими к результатам других групп ${ }^{11)}$, которые проводят численные симуляции на решетке для функциональных интегралов, определенных в импульсном представлении, а также решают подходящим образом усеченные УШД.

По нашему мнению, с физической точки зрения имеется по крайней мере два обстоятельства, которые можно отметить в связи с ИК-асимптотикой КХД.

Во-первых, следует помнить, что область $Q \lesssim 500$ МэВ физически отвечает расстояниям $r \gtrsim 10^{-13} \mathrm{~cm}$, т.е. адронным масштабам, на которых все понятия КХ Д, подобные

11) $\mathrm{C}$., например, работы [21], [23], [24] и короткое обсуждение различий их результатов в [14]. 
пропагаторам кварков и глюонов, по всей видимости, являются лишенными физического смысла. Даже в более “умеренной области” $0.5 \mathrm{MэB} Q \lesssim 3$ ГэВ, где ЭС КХД оказывается сильной, возникает вопрос о непосредственном физическом смысле непертурбативных функций КХД, включая эффективную функцию связи.

Результаты [21], [23], [24] для ЭС КХД, полученные недавно с помошью численных симуляций на решетке для функциональных интегралов, определенных в импульсном представлении, сформулированы на “обычном языке КТП" с использованием вершинных функций с фиксированной динамикой, как, например, в работах [23], [25] для модели КХД с двумя массивными кварками. В этих работах инвариантная функция связи $\bar{g}\left(Q^{2}\right)$ введена на основе глюон-кварковой вершины $\Gamma_{\mathrm{q}-\mathrm{gl}}\left(0 ; Q^{2}, Q^{2}\right)$ в частной МОМ-схеме при импульсе глюона, равном нулю. Определенная таким образом функция связи КХД имеет обычный для МОМ-схем недостаток - калибровочную зависимость. Тем не менее она может быть непосредственно соотнесена определенной физической ситуации (например, использована в разложении для наблюдаемой с калибровочно-зависимыми коэффициентами).

Во-вторых, уместно соотнести ИК-поведение функций КХД с явлением конфайнмента. Здесь можно обратиться к так назьваемому условию Куго-Оджима [26], отвечающему условию запрета на “открытый цвет” в асимптотических состояниях. В терминах КХД оно соответствует обрашению в нуль констант перенормировки глюонного и кварковых полей, что, в свою очередь, эквивалентно обрашению в нуль одетых пропагаторов этих полей в ИК-пределе. Такое поведение, вообще говоря, возможно при нулевом или конечном (см. работу [21]) ИК-пределе эффективной функции связи КХД. Как раз такое поведение было получено в большей части работ по решеточным симуляциям КХД [21], [23], [24]. Совсем недавно оно получило подтверждение в результатах группы Орсэ [27] на основе модели инстантонной жидкости, а также, в определенном смысле, в результатах анализа $\tau$-распада [28].

В этом контексте поведение (ALPHA-IRa) представляет вполне разумную возможность корреляции всех результатов решеточных симуляций друг с другом и с условием конфайнмента.

Благодарности. Автор признателен И.А. Арефьевой, В.С. Владимирову, Ю. Н. Дрожжинову, Б.И. Завьялову, М. Мюллеру-Пройсскеру, С.В. Михайлову, О. Пену и А.А. Славнову за полезные советы, а также А.В. Нестеренко за стимулирующие обсуждения и помощь в численных расчетах. Исследование было частично поддержано грантами РФФИ № 00-15-96691 и 02-01-00601, а также CERN INTAS № 99-0377.

\section{Список литературы}

[1] Н. Н. Боголюбов, Д. В. Ширков. ДАН СССР. 1955. Т. 103. С. 203; С. 391; ЖЭТФ. 1956. T. 30. C. 77 ; N. N. Bogoliubov, D. V. Shirkov. Nuovo Cimento. 1956. V. 3. P. 845

[2] Н. Н. Боголюбов, Д. В. Ширков. Введение в теорию квантованных полей. М.: Наука, 1984.

[3] D. Gross, F. Wilczek. Phys. Rev. Lett. 1973. V. 30. P. 1343.

[4] D. V. Shirkov. Toward the correlated analysis of perturbative QCD observables. Препринт ОИЯИ Е2-2000-46. Дубна: ОИЯИ, 2000; hep-ph/0003242.

[5] Д. В. Ширков. ТМФ. 2001. Т. 127. С. 3; hep-ph/0012283. 
[6] В. З. Бланк, В. Л. Бонч-Бруевич, Д. В. Ширков. ЖЭТФ. 1957. Т. 33. С. 265.

[7] P. A. M. Dirac. Theorie du positron. In: Septième Conseil de Physique Solvay: Structure et Propriété des Noyaux Atomiques (Oct. 22-29, 1933). Paris: Gauthier-Villars, 1934. P. 203.

[8] M. Lüscher, R. Narayanan, P. Weisz, U. Wolff. Nucl. Phys. B. 1992. V. 384. P. 168.

[9] ALPHA collaboration. Nucl. Phys. B. 1995. V. 437. P. 447; hep-lat/9411017.

[10] M. Lüscher. Advanced lattice QCD. In: Probing the Standard Model of Particle Interactions. Eds. R. Gupta et al. Amsterdam: Elsevier Sc., 1999. P. 229.

[11] A. Bode, R. Frezzotti, B. Gehrmann et al. Phys. Lett. B. 2001. V. 515. P. 49; hep-lat/0105003.

[12] J. Heitger, H. Simma, R. Sommer, U. Wolff. Nucl. Phys. B. Proc. Suppl. 2002. V. 106. P. 859; hep-lat/0110201.

[13] V. F. Kovalev. Acta Phys. Slovaca. 2002. V. 52. № 4. P. 353.

[14] Д. В. Ширжов. ТМФ. 2002. Т. 132. № 3. С. 484; hep-ph/0208082.

[15] K. A. Milton, I. L. Solovtsov. Phys. Rev. D. 1997. V. 55. P. 5295; hep-ph/9611438.

[16] В.С. Владимиров, Ю.Н. Дрожжинов, Б. И. Завьялов. Многомерные тауберовы теоремы для обобщенных функций. М.: Наука, 1986.

[17] А. Г. Постников. Тр. МИАН. 1979. Т. 144. С. 3.

[18] D. V. Shirkov, I. L. Solovtsov. Phys. Rev. Lett. 1997. V. 79. P. 1209; hep-ph/9704333.

[19] Г. Бәйтмен, А. Эрдейи. Таблицы интегральных преобразований. Т. 3. М.: Наука, 1969.

[20] Ю.А. Симонов. ЯФ. 1995. Т. 58. С. 113; А.М. Бадалян, Ю. А. Симонов. ЯФ. 1997. Т. 60. С. 714; Ю. А. Симонов. ЯФ. 2003. Т. 66. С. 796; hep-ph/0109159.

[21] L. Alkofer, L. von Smekal. Phys. Rep. 2001. V. 353. P. 281; hep-ph/0007355.

[22] E. Титчмари. Введение в теорию интегралов Фурье. М.: ОГИЗ, 1946.

[23] J. I. Skullerud, A. Kizilersu, A. G. Williams. Nucl. Phys. B. Proc. Suppl. 2002. V. 106. P. 841; hep-lat/0109027.

[24] Ph. Boucaud, J. P. Leroy, J. Micheli et al. Nucl. Phys. B. Proc. Suppl. 2002. V. 106. P. 266; hep-ph/0110171.

[25] J. Skullerud, A. Kizilersu. JHEP. 2002. V. 0209. P. 013; hep-ph/0205318.

[26] T. Kugo, I. Ojima. Progr. Theor. Phys. Suppl. 1979. V. 66. P. 1.

[27] Ph. Boucaud et al. JHEP. 2003. V. 0304. P. 005.

[28] S. L. Brodsky et al. Phys. Rev. D. 2003. V. 67. P. 055008; hep-ph/0212078.

Поступила в редакцию 2.XII.2002 г. 